\title{
Correction to: The Reform of the Orphan Drug Assessment System in China
}

\author{
Xiangyu Wang ${ }^{1,3} \cdot$ Lin Wang $^{2} \cdot$ Xiaoying Zheng $^{3,4} \cdot$ Qiang Zheng $^{5,6}$ \\ Published online: 2 March 2022 \\ ○) Springer Science+Business Media, LLC, part of Springer Nature 2022
}

\section{Correction to: Journal of Pharmaceutical Innovation https://doi.org/10.1007/s12247-021-09560-7}

In the original version of this article, published on 21 May 2021, unfortunately, the authors have found an error in the published version of the paper. Lead author Xiangyu Wang is affiliated with the following two institutions, which are listed as 1 and 3 in the published article:

1 Department of Science, Technology and International Cooperation, National Medical Product Administration, 100027, China.
3 Institute of Population Research, Peking University, Beijing, 100871, China.

Original article has been corrected.

Publisher's Note Springer Nature remains neutral with regard to jurisdictional claims in published maps and institutional affiliations.

The original article can be found online at https://doi.org/10.1007/ s12247-021-09560-7.

Xiaoying Zheng

xzheng@pku.edu.cn

$\triangle$ Qiang Zheng

zhengqiang@pku.edu.cn

1 Department of Science, Technology and International Cooperation, National Medical Product Administration, Beijing 100027, China

2 Department of Epidemiology, Bloomberg School of Public Health, John Hopkins University, Baltimore, MD 21202, USA

3 Institute of Population Research, Peking University, Beijing 100871, China

4 APEC Health Science Academy (HeSAY), Peking University, Beijing 100871, China

5 Department of Industrial Engineering and Management, College of Engineering, Peking University, Beijing 100871, China

6 Center for Pharmaceutical Information and Engineering Research, Peking University, Beijing 100871, China 